\title{
Sympathetic Renal Denervation for the Treatment of Hypertension: A Review of Current Progress, Limitations, Techniques, and the Future
}

\author{
Tushar Sharma*, Anjay Rastogi, Rishi Talwar, Reza Khosravani Goshtaseb, Rohit Kumar and \\ Mohamad Rashid
}

Research Department, Division of Nephrology, University of California, Los Angeles, USA

\begin{abstract}
Although the etiology of hypertension is complicated and multifactorial, the role of sympathetic overactivity as part of the pathogenesis in essential hypertension has been well described in the literature. A promising new treatment modality of Renal Sympathetic Denervation has become prominent with very enthusiastic support. This paper suggests that while the support may be overhyped, this new treatment modality may, with further research, become a powerful tool for a specific patient population. The history, benefits, and limitations of the procedure are discussed including, but not limited to, proper screening methodology, technical aspects, and procedural complications.
\end{abstract}

Keywords: Hypertension, refractory hypertension, secondary hypertension, sympathetic denervation.

\section{INTRODUCTION}

Essential or primary hypertension is a multifactorial disease. It is largely impossible to figure out a specific etiology for its development and therefore, it is equally difficult to identify a particular treatment modality that lowers blood pressure in all hypertensive patients. Nevertheless, the role of sympathetic nerves in its pathogenesis has been well described in the literature and so has the role of kidneys in the development of sympathetic over activity and hypertension. As a matter of fact, this led to the practice of surgical sympathectomy to treat hypertension as early as 1930-40s [1-3]. This practice had to be given up mainly due to high incidence of complications, like orthostatic hypotension and impotence, and the introduction of new anti-hypertensive medications [4]. Even though the procedure, by and large lost its significance, it underlined an important pathophysiological fact about hypertension, and paved the way for future research and interventions based on the concept.

\section{THE CURRENT NEED FOR EFFECTIVE THERAPY}

Due to the Simplicity HTN-1 and Simplicity HTN-2 trials, there has been a massive interest in this novel technique of Renal Sympathetic Denervation. Everyone, be it the physicians or the patients, has taken notice. In our view, the enthusiasm may not be misdirected, but it probably is overhyped. Amidst all the excitement, what seems to be lost, especially on the general population, is that, firstly it is not suitable for everyone. From the information that we have till now, a stringent protocol has to be followed in order to select the eligible patients, as we will discuss further.

*Address correspondence to this author at the Research Department, Division of Nephrology, University of California, Los Angeles, USA; Tel: 310-954-2692; Fax: 310-474-0725;

E-mail: RishiTalwar@ucla.edu
Secondly, as of now, this technique is not advocated to be a monotherapy. It is best used as an adjunct to the traditional medical treatment. Here, we propose a few considerations to be taken while screening patients for this procedure. Further along, we will discuss the technical aspects of the procedure in brief, with some of the complications that have been observed. At the end, we will mention some of the aspects future research should address, before this technique is unequivocally accepted as the milestone in hypertension therapy.

\section{SCREENING FOR ELIGIBILITY}

The aim of screening, as always is to select the patients who can undergo the procedure with minimum safety risk and maximum foreseeable benefits. Inappropriate use of renal denervation is of little benefit for the patients, puts them in unnecessary risk, and imposes an avoidable financial burden on healthcare. As such, the screening should ideally be done by a multidisciplinary team consisting of nephrologists, cardiologists and interventional radiologists. Patients $<18$ yrs or $>80$ yrs of age should be excluded, as should patients with serious comorbidities or any condition that the physicians think may endanger the wellbeing of the patient if he undergoes the procedure. Informed consent should be obtained from the patient after explaining the details of the procedure and the possible complications.

The next step is to determine whether the patient actually has "resistant hypertension". The American Heart Association defines resistant hypertension as a BP that remains above treatment goals despite the concurrent use of medications from three different antihypertensive classes, one ideally being a diuretic, with all agents prescribed at doses that provide optimal benefit. The importance of diuretics as antihypertensives cannot be stressed enough. They also prevent volume overload that may happen due to the concurrent use of vasodilators. The goal is $<140 / 90 \mathrm{~mm}$ $\mathrm{Hg}$ for general population and $<130 / 80$ for diabetes and 
chronic kidney disease. For the purpose of renal denervation procedure, a systolic BP above $160 \mathrm{~mm} \mathrm{Hg}$ has been the benchmark (150 in diabetics)and that is what we recommend as well $[5,6]$.

In daily clinical practice, inadequate or inappropriate doses of antihypertensive medications are common. Poor patient-doctor communication often leads to treatment inertia and noncompliance on the part of the patient. All these factors may cause what is called "pseudo resistance". Before a diagnosis of resistant hypertension is made, it is imperative to make sure that the patient has made the necessary life style changes, i.e. smoking cessation, weight loss, regular exercise and a balanced diet with low sodium and low fat consumption and a balanced potassium intake. Next, we should make sure that the patient is following an accurate and adequate pharmacological treatment. Combination therapies should be followed, including drugs acting on the Renin-Angiotensin-Aldosterone System (RAAS), diuretics, vasodilators and $\beta$-blockers. For the patients who cannot tolerate multi therapy and hence cannot control their BP, discretion is advised on the part of the physicians before considering them for renal denervation. For others, if the mentioned changes are implemented properly and the BP is still persistently high, a diagnosis of resistant hypertension may be considered, but not before excluding white coat effect (WCE, defined as a difference between office BP and daytime ambulatory BP $>20 \mathrm{~mm} \mathrm{Hg} \mathrm{SBP}$ and/or $>10 \mathrm{~mm}$ $\mathrm{Hg}$ DBP). The best way to rule out White coat hypertension is to do a 24 hour Ambulatory BP monitoring (ABPM). ABPM is also recommended in the screening before renal denervation in the European Society of Hypertension (ESH) position paper [7]. It is an inexpensive test and it is expected to rule out a considerable number of patients from more expensive and cumbersome screening procedures [8].

Our next target should be to exclude secondary forms of hypertension, such as primary aldosteronism and pheochromocytoma (Fig. 1). It becomes more important if we consider the fact that in both Simplicity HTN-1 and HTN-2 trials, secondary hypertension hasn't been excluded very well. A significant number of patients suspected to have resistant essential hypertension actually have some form of secondary hypertension [9-11]. These forms are unlikely to respond to renal sympathetic denervation, since overactive sympathetic drive does not play a very important role in the pathogenesis of these disease pathways. Primary aldosteronism, for example, causes a volume dependent hypertension that is characterized by a decreased sympathetic activity. The best method to diagnose primary aldosteronism is the aldosterone: renin ratio, after all the medications interfering with the RAAS have been temporarily stopped. Pheochromocytoma can be diagnosed by elevated metanephrine levels in 24 hour urine samples. Other etiologies for secondary hypertension need to be excluded accordingly. The group of patients that we now have, after excluding secondary causes of hypertension, can be considered to have resistant essential hypertension, and they may potentially benefit from renal denervation.

Finally, the renal artery anatomy needs to be studied to ascertain if it is suitable for the procedure. Renal imaging is probably best done with magnetic resonance angiography, since it gives excellent pictures without radiation exposure. Besides, it uses a contrast agent similar to gadolinium, that can be used safely even in patients with kidney disease. If MRA cannot be done, CT angiography or Doppler duplex sonography is acceptable alternatives. Nevertheless, the amount of contrast should be monitored closely, particularly in patients with kidney failure. The renal artery should be $>20 \mathrm{~mm}$ long and diameter should be $>4 \mathrm{~mm}$. Previously required stenting or evidences of renal artery stenosis are contraindications for the procedure. Initially, renal denervation was contraindicated in patients with $\mathrm{eGFR}<45 / \mathrm{ml} / \mathrm{min} / 1.73 \mathrm{~m}^{2}$ because of theoretical possibility

\begin{tabular}{|c|c|c|}
\hline $\begin{array}{l}\text { Secondary Causes of } \\
\text { Hypertension }\end{array}$ & Signs and Symptoms & $\begin{array}{c}\text { Estimated Prevalence Amongst } \\
\text { Hypertensive Patients }\end{array}$ \\
\hline Renal parenchymal disease & Nocturia, Edema & $1-8 \%$ \\
\hline Hyper-aldosteronism & Fatigue, hypokalemia & $5-15 \%$ \\
\hline Renal artery disease & $\begin{array}{c}\text { Severe hypertension In very young or old } \\
\text { patients, + Hx of smoking, vascular disease, } \\
\text { on multiple HTN medications, abdominal } \\
\text { bruit }\end{array}$ & $3-4 \%$ \\
\hline Hyper or hypothyroidism & Weight loss(gain)anxiety(fatigue) & $1-3 \%$ \\
\hline Cushing 's Syndrome & Striae, Weakness, fluid retention, obesity & $1-5 \%$ \\
\hline Pheochromocytoma & $\begin{array}{c}\text { Headache, diaphoresis, hypertension } \\
\text { attacks }\end{array}$ & $1-5 \%$ \\
\hline Coarctation of aorta & $\begin{array}{l}\text { Different systolic blood pressure in upper } \\
\text { and lower extremities }\end{array}$ & $1.0 \%$ \\
\hline
\end{tabular}

Fig. (1). 
of damage to the kidney vasculature by this technique. However, recent studies have shown that it is safe to perform this procedure in patients who have an eGFR lower than previously recommended levels $[12,13]$. Renal denervation does not appear to aggravate functional or structural renal damage and may be safe in progressive kidney disease. This makes sense, keeping in mind that patients with renal failure have an increased amount of sympathetic activity compared to hypertensive patients who have normal kidney function $[14,15]$. Similarly, in the initial studies, presence of accessory renal arteries was considered as a contraindication, but several new studies are including such patients, given that the arteries are $>4 \mathrm{~mm}$ in diameter and $>20 \mathrm{~mm}$ in length $[13,16,17]$.

\section{TECHNICAL APPROACH}

The procedure is carried out by experienced vascular interventionists. The femoral artery access point is prepared and local anesthesia is administered. The procedure is carried out under conscious sedation or under general anesthesia. The baseline activated clotting time (ACT) is determined. A $6 \mathrm{~F}$ guiding catheter is placed through the access and its tip is advanced into the renal artery. Angiography is performed followed by administration of nifedipine or nitroglycerine for spasm prophylaxis and heparin for thrombosis prophylaxis. When an ACT of 25-300 is achieved, the Simplicity catheter (Ardian-Medtronic, Minneapolis, Minnesota) is advanced to a position just proximal to the bifurcation of the renal artery, and brought into contact with the endothelium. When the impedance is stable, radiofrequency energy of $8 \mathrm{~W}$ is applied, with a maximum temperature of $70^{\circ}$ C. During each emission of radiofrequency, the impedance, temperature and power are continuously monitored according to a predetermined algorithm. The treatment is interrupted if any of these 3 deviates from the algorithm. Slight modification of the catheter position may be required, if the impedance is too high. After every treatment point, each lasting 2 minutes, the catheter is pulled back $5 \mathrm{~mm}$, rotated anticlockwise and another radiofrequency ablation is done. This sequence is continued for 4-6 times, thus creating a spiral pattern of ablation. The same procedure is then repeated on the contralateral renal artery. After the entire procedure is completed, typically after about an hour, the access site can be closed with sutures or by manual compression of the puncture site. The patient is advised to remain supine for 24 hours, keeping the leg immobile and straight. Depending on the patient, the hospitalization is typically for 1-2 days.

Another catheter has been developed by St Jude Medical, St Paul, USA, that has been used in the EnligHTN clinical trials in Europe and Australia. The basic technique is the same with some minor changes. It is a multi-electrode basket catheter that has 4 electrodes. Once put in a position, radiofrequency energy is given for 90 seconds through each electrode sequentially. Then the basket is collapsed and pulled back $1 \mathrm{~cm}$, where the same sequence is repeated. The remainder of the procedure is essentially the same. This catheter has been shown to reduce the procedure time to 38 minutes. Also, it is said to produce more reproducible effects, since the ablation is more organized and consistent.

\section{PROCEDURAL COMPLICATIONS}

In the Simplicity HTN-1 trial, the reported procedural complications were one renal artery dissection, requiring renal artery stenting, three inguinal pseudoaneurysms, and four cases of post-procedural temporary flank pain. In HTN2 trial, one case each of pseudoaneurysm, post-therapeutic hypotension, temporary paraesthesia and flank pain was reported. In other studies, the reported complications include severe arterial spasm, imminent respiratory and cardiocirculatory depression in a patient with OSA and bradycardia, among others [16,18]. Not to forget, the radiation exposure in this procedure may be significant, especially as the eligible population may have many obese patients who require higher amounts of radiation. It is also worth mentioning here that the incidence of the mentioned complications has been few and far in between. Nonetheless, it is prudent to exercise caution with the procedure and be sure it is being used on a targeted and specific patient population.

Recent assessments done by optical coherence tomography show presence of edema, vasospasm, endothelial or intimal disruptions and intraluminal thrombus at the site of ablation [19]. This study notes that the EnligHTN multi-electrode basket causes a different type of tissue injury from the Simplicity catheter, and apparently leads to a higher amount of intraluminal thrombus formation. This certainly requires a long term follow up in larger studies to assess long term histological impact on the renal arteries due to the procedure.

\section{CLINICAL TRIALS AND DATA}

Some promising data regarding Renal Sympathetic Denervation has arisen out of the Simplicity HTN-1 and the Simplicity HTN-2 trials. In the HTN-2 trial, 51 patients were assigned as controls while 49 underwent RDN with the Simplicity device catheter and both groups continued their antihypertensive medications. At six months, the RDN therapy plus antihypertensive medication arm had a reduction in mean blood pressure of $(-32 /-12 \mathrm{mmHg})$ while the control group receiving only medications had little to no change in baseline blood pressures $(+1 / 0 \mathrm{mmHg})$ [6]. In March 2013, the data from the 24 month follow up of all Simplicity patient subjects was presented at the American Cardiology Congress. The original RDN treatment arm, the control crossover arm (the control group that underwent RDN after 6 months), and the combined cohort of all achieved sustained blood pressure reductions of $(-29 /-10$ $\mathrm{mmHg}),(-35 /-13 \mathrm{mmHg})$, and $(-31 /-11 \mathrm{mmHg})$ respectively [20]. There were no serious procedure-related events in either Symplicty HTN-1 or Symplicity HTN-2 clinical trials and renal function was maintained. A third clinical trial titled Symplicity HTN-3 has been designed and is currently ongoing where both the study patients and the research staff who obtain the blood pressure measurements will be blinded. It is a prospective, randomized, masked procedure, singleblind trial. It aims to randomize 530 patients to RDN and control groups in a 2:1 ratio [21]. Furthermore, in order to obtain a clearer picture of the blood pressure variations before and after the procedure, the change in average 24hour ambulatory blood pressure will be a secondary outcome measure [22]. 
Another study by Brandt et al. was designed to particularly examine the effects of RDN on clinical hard endpoints like left ventricular hypertrophy. Using 18 patients as the control group, 46 patients underwent bilateral sympathetic denervation. Transthoracic echocardiograms were performed at baseline, 1 month post-procedure, and 6 months post-procedure. While there were no significant changes recorded in the control group, the RDN arm had the following results recorded: A reduction of systolic and diastolic blood pressure (-22.5/-7.2 $\mathrm{mm} \mathrm{Hg}$ at 1 month and $-27.8 /-8.8 \mathrm{~mm}$ Hgat 6 months, $\mathrm{p}<0.001$ at each time point). A reduction in mean interventricular septum thickness from $14.1 \pm 1.9 \mathrm{~mm}$ to $13.4 \pm 2.1 \mathrm{~mm}$ and $12.5 \pm 1.4 \mathrm{~mm}$ $(\mathrm{p}<0.007)$, and LV mass index from $53.9 \pm 15.6 \mathrm{~g} / \mathrm{m}(2.7)$ $(112.4 \pm 33.9 \mathrm{~g} / \mathrm{m} 2)$ to $47.0 \pm 14.2 \mathrm{~g} / \mathrm{m}(2.7)(103.6 \pm$ $30.5 \mathrm{~g} / \mathrm{m} 2)$ and $44.7 \pm 14.9 \mathrm{~g} / \mathrm{m}(2.7)(94.9 \pm 29.8 \mathrm{~g} / \mathrm{m} 2)$ $(\mathrm{p}<0.001)$ at 1 month and 6 months, respectively [23] was observed.

A study by Witkowski et al. in Poland examined the changes in sleep apnea and glycemic control in a group of 10 patients with resistant hypertension and sleep apnea who underwent RDN. The patients underwent 3 and 6 month follow ups with blood chemistry analyses and polysomnography investigations. In addition to decreases in plasma glucose concentration 2 hours after glucose administration (median: 7.0 versus $6.4 \mathrm{mmol} / \mathrm{L}$; p <0.05), there were reductions observed in hemoglobin A1C levels (median: $6.1 \%$ versus $5.6 \%$; $<0.05$ ) at 6 months. There was also a decrease in apnea-hypopnea index at 6 months after renal denervation (median: 16.3 versus 4.5 events per hour; $p$ $<0.059)$. The researchers concluded that RDN lowered mean BP in patients with resistant hypertension and sleep apnea, reduced severity of sleep apnea, and improved glucose tolerance [24].

\section{DISCUSSION}

The novel technique of renal denervation has given a new hope to the patients and physicians alike. But the process requires caution and expertise at every step, starting from meticulous selection of eligible patients to the technical approach of the procedure and all the way to the long term follow up. Analysis of patients who underwent the procedure and had positive results should be compared to those groups which had more neutral or varied outcomes. This can be a possible avenue for determining criteria that would better provide us a prediction of the outcome of the procedure. For example, patients who have high blood pressure especially before the procedure may have greater reduction in average $\mathrm{BP}$ after the procedure than patients with typical resistant hypertension. Perhaps the age of onset and/or degree of sympathetic drive as part of the pathogenesis of a specific patient's hypertension could be determined and used as a predictive criterion.

There also exists a desire for intraprocedural verification of ablation. Currently, the procedure is a blind process, in which the results are seen long after the patient is off the table. A method of intraprocedural verification of ablation would not only lower the costs, but also benefit the interventionists and patients alike. Recent discussion has also given rise to the topic of increased number of ablation sites. Large multicenter and systematic research is needed regarding this issue in order to properly weigh the possible benefits with the risk of complications [25]. The difference in anatomy of renal arteries of patients selected for the procedure requires further research as well. For example, the relationship between the artery wall thickness and response of BP from the denervation procedure is of interest [18].

Although there has been success as of late with the pinpoint BP measurements as the endpoints of the procedure, there has been discussion of possibly implementing Ambulatory Blood Pressure Monitoring. This would serve as a more comprehensively accurate goal endpoint and measure of success. There is also lack of a systematic long term histological follow-up of the patients who have undergone the procedure. Optical coherence tomography has been used successfully in the past and this may a good option for this type of specific monitoring.

\section{CONCLUSION}

Sympathetic Renal Denervation is a novel and potentially powerful method of treatment for hypertension for that selected group of patients who have been found to have elevated blood pressures refractory to standard treatment modalities. However, there still remains much to be learned and studied about this procedure in order to optimize its use with the least amount of procedural complications and to reduce expense by using it in patients who would benefit the most from the procedure. It is necessary to ensure patients have genuine resistant primary hypertension and are not suffering from the many secondary causes of hypertension. Furthermore, large, randomized, controlled, multicenter studies are necessary to further validate the safety and efficacy of sympathetic renal denervation in specific resistant hypertension patient populations who have Chronic Kidney Disease. Lastly, although evidence of significant damage to renal functioning and the endothelium is not yet present, long-term monitoring after the procedure is required.

\section{CONFLICT OF INTEREST}

The authors confirm that this article content has no conflict of interest.

\section{ACKNOWLEDGEMENTS}

Declared none.

\section{REFERENCES}

[1] Grimson KS. Total thoracic and partial to total lumbar sympathectomy and celiac ganglionectomy in the treatment of hypertension. Ann Surg [Internet]. 1941 Oct [cited 2013 Jul 19]; 114 (4): 753-75. Available from: http://www.pubmedcentral.nih. gov/articlerender.fcgi?artid=1385824\&tool=pmcentrez\&rendertype =abstract

[2] Peet MM. Results of bilateral supradiaphragmatic splanchnicectomy for arterial hypertension. N Eng J Med [Internet]. 1947 236(8): 270-7.

[3] Grimson KS, Orgain ES, Anderson B, D’Angelo GJ. Total thoracic and partial to total lumbar sympathectomy, splanchnicectomy and celiac ganglionectomy for hypertension. Ann Surg 1953 138(4): 532-47.

[4] Whitelaw GP, Kinsey D, Smithwick RH. Factors influencing the choice of treatment in essential hypertension. Surgical, Medical or a combination of both. Am J Surg 1964; 107: 220-31.

[5] Krum H, Schlaich M, Whitbourn R, et al. Catheter-based renal sympathetic denervation for resistant hypertension: a multicentre safety and proof-of-principle cohort study. Lancet 2009; 373(9671): 1275-81. 
[6] Esler MD, Krum H, Sobotka PA, et al. Renal sympathetic denervation in patients with treatment-resistant hypertension (The Symplicity HTN-2 Trial): a randomised controlled trial. Lancet 2013; 376(9756): 1903-9.

[7] Schmieder RE, Redon J, Grassi G, et al. ESH position paper: renal denervation - an interventional therapy of resistant hypertension. J Hypertens 2012; 30(5): 837-41.

[8] Banegas JR, Messerli FH, Waeber B, et al. Discrepancies between office and ambulatory blood pressure: clinical implications. Am J Med 2009; 122(12): 1136-41.

[9] Omura M, Saito J, Yamaguchi K, Kakuta Y, Nishikawa T. Prospective study on the prevalence of secondary hypertension among hypertensive patients visiting a general outpatient clinic in Japan. Hypertens Research 2004; 27(3): 193-202.

[10] Sukor N. Endocrine hypertension--current understanding and comprehensive management review. Eur J Intern Med 2011; 22(5): 433-40.

[11] Viera AJ, Neutze DM. Diagnosis of secondary hypertension: an age-based approach. Am Fam Phys 2010; 82(12): 1471-8.

[12] Hering D, Mahfoud F, Walton AS, et al. Renal denervation in moderate to severe CKD. J Am Soc Nephrol 2012; 23(7): 1250-7.

[13] Verloop WL, Vink EE, Voskuil M, et al. Eligibility for percutaneous renal denervation: the importance of a systematic screening. J hyperten [Internet]. 2013; 31(8): 1662-8.

[14] Vink EE, Blankestijn PJ. Evidence and consequences of the central role of the kidneys in the pathophysiology of sympathetic hyperactivity. Front Physiol 2012; 3: 29.

[15] Blankestijn PJ, Ritz E. Renal denervation: potential impact on hypertension in kidney disease? Nephrology, dialysis, transplantation: official publication of the European dialysis and Transplant Association - European Renal Association 2011; 26(9): 2732-4.

[16] Scheurig-Muenkler C, Weiss W, Foert E, et al. Renal Denervation for Refractory Hypertension - Technical Aspects, Complications and Radiation Exposure. RoFo : Fortschritte auf dem Gebiete der Rontgenstrahlen und der Nuklearmedizin 2013; 185(6): 550-7.
[17] Damascelli B, Patelli G, Tichá V, et al. Catheter-based radiofrequency renal sympathetic denervation for resistant hypertension. J Vasc Interven Radiol 2013; 24(5): 632-9.

[18] Dahm JB, Wolpers H-G, Becker J, Daume B, Hansen C. [Renal sympathetic denervation--physical-antomical basics, procedure, and analysis of clinical and technical parameters on procedure success and efficacy]. Deutsche medizinische Wochenschrift 2012; 137(39): 1904-9.

[19] Templin C, Jaguszewski M, Ghadri JR, et al. Vascular lesions induced by renal nerve ablation as assessed by optical coherence tomography: pre- and post-procedural comparison with the Simplicity(R) catheter system and the EnligHTN ${ }^{\mathrm{TM}}$ multi-electrode renal denervation catheter. Eur Heart J 2013; Available from: http://www.ncbi.nlm.nih.gov/pubmed/23620498

[20] Esler MD, Krum H, Schlaich M, Schmieder RE, Böhm M, Sobotka PA. Renal sympathetic denervation for treatment of drug-resistant hypertension: one-year results from the Symplicity HTN-2 randomized, controlled trial. Circulation [Internet]. 2012; 126(25): 2976-82.

[21] Kandzari DE, Bhatt DL, Sobotka PA, et al. Catheter-based renal denervation for resistant hypertension: rationale and design of the SYMPLICITY HTN-3 Trial. Clin Cardiol 2012; 35(9): 528-35.

[22] ClinicalTrials.gov. Renal denervation in patients with uncontrolled hypertension (SYMPLICITY HTN-3) [Internet]. Available from: http://clinicaltrials.gov/ct2/show/NCT01418261

[23] Brandt MC, Reda S, Mahfoud F, Lenski M, Böhm M, Hoppe UC. Effects of renal sympathetic denervation on arterial stiffness and central hemodynamics in patients with resistant hypertension. J Am Coll Cardiol 2012; 60(19): 1956-65.

[24] Witkowski A, Prejbisz A, Florczak E, et al. Effects of renal sympathetic denervation on blood pressure, sleep apnea course, and glycemic control in patients with resistant hypertension and sleep apnea. Hypertension 2011; 58(4): 559-65.

[25] Xiao Y, Zhou S, Liu Q. Ablation points of renal sympathetic denervation: the more, the better? Hypertension 2012; 60(6): e47.

(c) Sharma et al.; Licensee Bentham Open.

This is an open access article licensed under the terms of the Creative Commons Attribution Non-Commercial License (http://creativecommons.org/licenses/by-nc/3.0/) which permits unrestricted, non-commercial use, distribution and reproduction in any medium, provided the work is properly cited. 